\title{
Reaching for the Holy Grail: insights from infection/cure models on the prospects for vaccines for Trypanosoma cruzi infection
}

\author{
Juan Bustamante', Rick Tarleton²/+ \\ 'ISGlobal-Instituto de Salud Global de Barcelona, Centro de Investigación en Salud Internacional de Barcelona, Hospital Clínic, \\ Universitat de Barcelona, Barcelona, Spain ${ }^{2}$ Center for Tropical and Emerging Global Diseases, University of Georgia, Athens, GA, USA
}

Prevention of Trypanosoma cruzi infection in mammals likely depends on either prevention of the invading trypomastigotes from infecting host cells or the rapid recognition and killing of the newly infected cells by T. cruzispecific T cells. We show here that multiple rounds of infection and cure (by drug therapy) fails to protect mice from reinfection, despite the generation of potent $T$ cell responses. This disappointing result is similar to that obtained with many other vaccine protocols used in attempts to protect animals from T. cruzi infection. We have previously shown that immune recognition of $\mathrm{T}$. cruzi infection is significantly delayed both at the systemic level and at the level of the infected host cell. The systemic delay appears to be the result of a stealth infection process that fails to trigger substantial innate recognition mechanisms while the delay at the cellular level is related to the immunodominance of highly variable gene family proteins, in particular those of the trans-sialidase family. Here we discuss how these previous studies and the new findings herein impact our thoughts on the potential of prophylactic vaccination to serve a productive role in the prevention of $\mathrm{T}$. cruzi infection and Chagas disease.

Key words: Chagas disease - vaccine - Trypanosoma cruzi

Vaccination to a wide range of bacterial and viral infections has without question saved 100's of millions of lives and many billions of dollars in treatment costs and lost productivity (Rappuoli et al. 2014). So it is expected that the ultimate goal for immunological studies into any animal pathogen is the development of a highly effective prophylactic vaccine. Unfortunately such vaccines for human parasitic diseases have as yet alluded researchers. This is not for lack of effort in most cases. With respect to T. cruzi, the agent of human Chagas disease, there have been considerable efforts applying a broad range of technologies [reviewed in (QuijanoHernandez \& Dumonteil 2011, Vazquez-Chagoyan et al. 2011)]. Many vaccines have been shown to increase the rate and quality of the infection control in animal models, but none have actually prevented the establishment of the infection. In effect, these vaccines do no better than what the natural immune system ultimately does on its own - establish very good pathogen control, but rarely elimination of the infection. And because it is persistent infection rather than acute lethality that is by far the primary cause of morbidity and mortality in Chagas disease, these partially effective vaccines would seem to have rather limited utility, particularly for preventing human disease. Herein we consider reasons why this is the case with $T$. cruzi infection and Chagas disease and

doi: 10.1590/0074-02760140440

Financial support: NIH-USA

+ Corresponding author: tarleton@uga.edu

Received 20 November 2014

Accepted 25 February 2015 consider whether there might be diseases for which vaccines will remain elusive, irrespective of the knowledge and technologies brought to bear.

A number of conditions have to be met and information known in order to assess the potential for an effective prophylactic/sterilising vaccine. Among these are (i) Are the mechanisms of immune control understood? (ii) Does the normal response to infection at least occasionally result in parasitological cure? and (iii) Are those who resolve/cure the infection resistant to reinfection?

Addressing these questions, there is a reasonably clear understanding of the immune effector mechanism that are particularly important in immune control of $T$. cruzi (Tarleton 2007). Whether these responses can achieve parasitological cure, the answer for T. cruzi appears to be yes - both in humans and in animal models "spontaneous cure" of infection (e.g. complete parasite clearance without chemical or immunological interventions) does occur (Francolino et al. 2003, Dias et al. 2008, Bertocchi et al. 2013, Tarleton 2013). However such cures take a considerable period of time and are also relatively rare. Thus, although the standard infection with $T$. cruzi is life-long, with low parasite burden, occasionally the balance tips toward complete parasite elimination. It is not clear why some infections are cured while most are not and the immunological correlates of these cures are not known. It may simply be that this is a war of attrition, with the host occasionally prevailing outright. For a vaccine to be effective in T. cruzi infection it has to force the immune system to routinely and dependably wage a more effective battle without generating greater damage. Without better knowledge of the immunological correlates of cure, this may be difficult to achieve.

Because spontaneous cures are rare, determining whether hosts that completely resolve the infection are resistant to reinfection has not been previously investi- 
gated. As part of this study we addressed this issue by assessing immune protection acquired in mice cured of infection using benznidazole (BZ) treatment.

\section{MATERIALS AND METHODS}

Mice, parasites and infections - C57BL/6 (Ly5.2+) (B6) mice were purchased from National Cancer Institute at Frederick (USA) and were maintained in the University of Georgia animal facility in microisolator cages under specific pathogen-free conditions. Tissue culture trypomastigotes (TCT) of the CL strain of T. cruzi were obtained from passage through Vero cells. Mice were infected intraperitoneally (i.p.) with 1,000 Brazil strain TCT and sacrificed by $\mathrm{CO}_{2}$ inhalation at different time points post-infection. Mice were reinfected i.p with $10^{3}$ TCT of T. cruzi CL strain at different time points postinfection. In some experiments mice were challenged in the hind foot pads with $2.5 \times 10^{5} \mathrm{~T}$. cruzi tdTomato trypomastigotes as described previously and the fluorescent intensity as a surrogate of parasite load was measured using a whole animal imaging system (Maestro2 In Vivo Imaging System CRi, USA) (Canavaci et al. 2010).

$B Z$ treatment - N-Benzyl-2-nitroimidazole acetamide (benznidazole; Rochagan, Roche, Brazil) was used as a trypanocidal drug. Mice were treated orally with daily doses of BZ of $100 \mathrm{mg} / \mathrm{kg}$ of body weight for 40 days [15-55 days post-infection (dpi)]. BZ was prepared by pulverisation of one tablet containing $100 \mathrm{mg}$ of the active principle, followed by suspension in distilled water. Each mouse received $0.20 \mathrm{~mL}$ of this suspension by gavage.

Assessment of treatment efficacy - Mice were immunosuppressed with cyclophosphamide $(200 \mathrm{mg} / \mathrm{kg} /$ day $)$ i.p. at two-three day intervals for a total of four doses. Following immunosuppression, blood was collected via tail vein and the number of parasites was quantified using a Neubauer haemocytometer. Survival was monitored daily. The DNA preparation, generation of polymerase chain reaction (PCR) standards and detection of parasite tissue load by real-time PCR was carried out as described previously (Cummings et al. 2003, Bustamante et al. 2008). Skeletal, heart and fat tissues were collected at various time points post-treatment and fixed in $10 \%$ buffered formalin. Sections $(5 \mu \mathrm{m})$ from paraffin-embedded tissues were stained with haematoxylin and eosin for histopathological analysis.

$T$ cell phenotyping - Red blood cells (RBCs) in single cell suspensions of spleen cells (SC) were lysed in a hypotonic ammonium chloride solution and washed in staining buffer $\{2 \%$ bovine serum albumin, $0.02 \%$ azide in phosphate buffered saline (PBS) [PAB] .

In some cases, mouse peripheral blood was obtained by retro-orbital venipuncture, collected in Na citrate solution and washed in PAB. Whole blood was incubated with tetramer-PE and the following labelled Abs: antiCD44 FITC, anti-KLRG1 PECy7, anti-CD8 EFluor 450, anti-CD127 APC (eBioscience, USA). Cells were also stained with anti-CD4, anti-CD11b and anti-B220 (Caltag-Invitrogen Laboratories, USA) for use as an exclusion channel. Cells were stained for $45 \mathrm{~min}$ at $4^{\circ} \mathrm{C}$ in the dark, washed twice in PAB and fixed in $2 \%$ formaldehyde.
RBCs were lysed in a hypotonic ammonium chloride solution after washing twice in PAB. At least 500,000 cells were acquired using a Cyan flow cytometer (DakoCytomation, USA) and analysed with FlowJo software (Tree Star Inc, USA). MHC I tetramers TSKB20 (ANYKFTLV/K ${ }^{\mathrm{b}}$ ) was synthesised at the Tetramer Core Facility (Emory University, USA).

Intracellular cytokine staining - SC from naïve, untreated/chronic or treated/cured mice were stimulated with T. cruzi peptides $(5 \mu \mathrm{M})$ at $37^{\circ} \mathrm{C}$ for $5 \mathrm{~h}$ in the presence of Brefeldin A (GolgiPlug; BD Pharmingen, USA). T. cruzi peptides used in this study were TSKB20 (ANYKFTLV) and TSKB74 (VNYDFTLV) (Martin et al. 2006). Cells were surface stained with anti-CD8 EFluor 450 (eBioscience) and intracellular cytokine staining was performed with anti-interferon- $\gamma$ APC and with a Cytofix/Ctyoperm kit (BD Biosciences, USA) in accordance with the manufacturer's instructions. At least 250,000 lymphocyte events were acquired and analysed as above.

In vivo cytotoxicity assay - SC from naïve mice were incubated either with the T. cruzi peptide TSKB20 (ANYKFTLV), TSKB74 (VNYDFTLV) or with no peptide for $1 \mathrm{~h}$ at $37^{\circ} \mathrm{C}$. Cells were washed twice with PBS and incubated with $2.5 \mu \mathrm{M}$ CFSE (CFSE ${ }^{\text {high }}$ ) for TSKB20 peptide-loaded cells, $1.0 \mu \mathrm{M}$ CFSE $\left(\right.$ CFSE $\left.^{\text {int }}\right)$, for TSKB74 peptide-loaded cells or $0.25 \mu \mathrm{M}$ CFSE (CF$\mathrm{SEl}^{\text {ow }}$ ) for unpulsed cells for $3 \mathrm{~min}$ at room temperature. The CFSE was quenched with FBS and the CFSE ${ }^{\text {low }}$ and CFSE $^{\text {high }}$ cells were combined and transferred intravenously into naïve, untreated/chronic and treated/cured mice. SCs were harvested after $16 \mathrm{~h}$ and $\mathrm{CFSE}^{\text {high }}, \mathrm{CF}-$ $\mathrm{SE}^{\text {int }}$ and CFSE ${ }^{\text {low }}$ cells were detected by flow cytometry. The percentage of specific killing was determined using the formula: $\left\{1-\left[\left(\% \mathrm{CFSE}^{\text {low }}\right.\right.\right.$ naïve $/ \% \mathrm{CFSE}^{\text {high }}$ naïve $) /$ (\% $\% \mathrm{CFSE}^{\text {low }}$ chronic/\%CFSE ${ }^{\text {high }}$ chronic) $\left.]\right\} 100 \%$.

Ethics - All animal protocols were approved by the University of Georgia Institutional Animal Care and Use Committee.

\section{RESULTS AND DISCUSSION}

Effective vaccines faithfully mimic the immune protection afforded by survival of a full-fledged infection. Since complete spontaneous clearance of T. cruzi infection is rare, we simulated the immunity induced by resolution of infection by curing established infections using BZ treatment. Our previous studies have established the conditions for achieving and methods to monitor parasitological cure using this approach (Bustamante et al. 2008). In initial experiments, mice were infected with $10^{3}$ trypomastigotes and BZ treatment was initiated on day 15 post-treatment and then challenge infection was delivered $\sim 150$ days after the completion of drug treatment (Fig. 1A). Mice were then immunosuppressed $\sim 45$ days after challenge to reveal persistence of infection as assessed by parasites in blood and tissues. Previous studies have established this immunosuppression protocol as a standard for detecting persistent infection and for determining the curative potential of candidate drugs (Bustamante et al. 2008, 2014). As shown in Fig. 1B, treated but not re-challenged mice were free of infection, demonstrating 
A

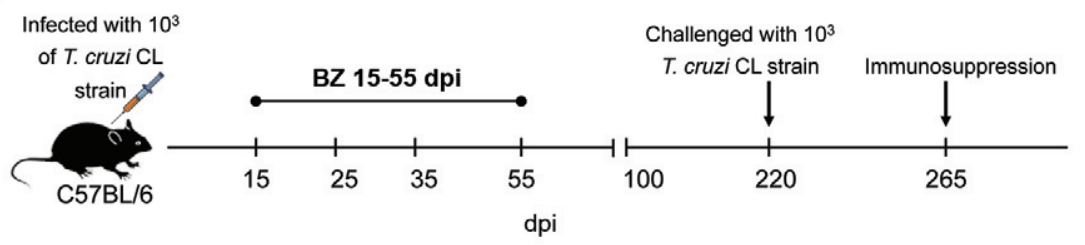

B

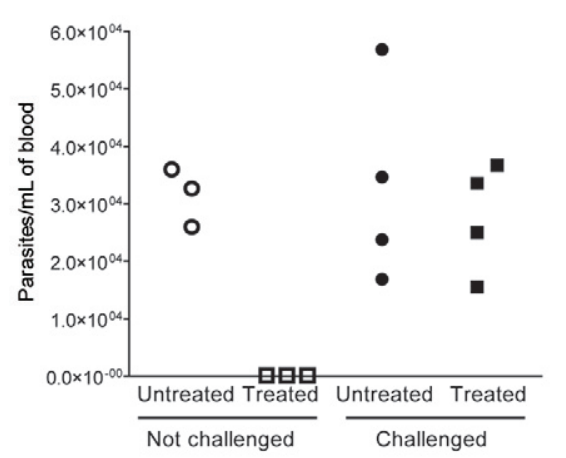

C

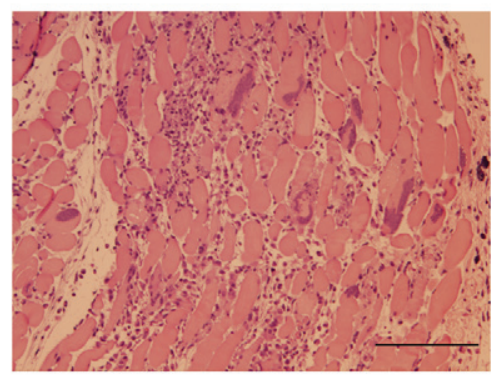

Fig. 1: Trypanosoma cruzi-infected and cured mice are not sterilely immune to reinfection. A: schematic representation of infection, treatment, reinfection and immunosuppression. Mice infected for 15 days with $10^{3}$ trypomastigotes of the $T$. cruzi CL strain were left untreated or were treated with a 40 day course of benznidazole (BZ) at $100 \mathrm{mg} / \mathrm{kg} /$ day. Mice were there challenge infected with the same strain/dose of $T$. cruzi or not challenged and then 45 days later (day 265 of the experiment) immunosuppressed with cyclophosphamide as previously described (Bustamante et al. 2008); B: detection of parasites in blood or muscle tissue (C) two weeks after immunosuppression indicates the failure of infection/cure protocol to protect mice from challenge infection. Treated but not-re-challenged mice had no detectable parasitaemia following immunosuppression (B) indicating the ability of the BZ treatment to provide parasitological cure; C: histological sections of the skeletal muscle at 280 days post-infection (dpi). Presence of absence of tissue parasites was confirmed by quantitative polymerase chain reaction (data not shown). Bar $=200 \mu \mathrm{m}$.

again the ability of this BZ treatment regimen to achieve parasitological cure. However both persistently infected (untreated) and infected/cured mice (treated) exhibited parasites in the blood and in tissues following re-challenge (Fig. 1C). Thus, mice cured of T. cruzi infection are not resistant to reinfection with a low dose of T. cruzi.

To follow the reinfection process at the infection site more closely, we assessed initial protection using fluorescent protein-expressing parasites delivered into the footpads of single or multiple dose "immunised" as previously described (Collins et al. 2011) (Fig. 2A). As predicted, a single round of infection and cure provided essentially no protection based on the footpad infection model (Fig. 2B). In contrast, similar challenge infection in continuously infected mice (not BZ-treated group) resulted in more rapid control of the footpad challenge relative to either noninfected or infected/cured mice.

We then asked if boosting of the immune protection could be achieved by multiple rounds of infection and cure. Attainment of parasitological cure after each round of infection and treatment was confirmed by detection of predominantly CD127-expressing, T central memory phenotype $T$. cruzi-specific T cells in the blood of mice (Fig. 3A). Continuously infected mice maintained a relatively stable and low level of CD127-expressing T cells specific for the immunodominant TSKb20 T. cruzi transsialidase (ts)-derived epitope whereas drug-cured mice exhibited a decrease in the overall number of TSKb20specific $\mathrm{T}$ cells and an increasing proportion of CD127 expression, indicative of the absence of continuous antigen stimulation in these cured mice (Bustamante et al. 2008) (Fig. 3A, B). Multiple rounds of infection and cure increased the ability of mice to control initial parasite growth in the footpad, with three rounds of infection and cure providing a level of protection nearly equivalent to that afforded by continuous infection (Fig. 2B-G).

Even nonvaccinated mice ultimately control T. cruzi at the initial infection site in the footpad resulting in the absence of detectable parasites by immunofluorescence at $>2$ weeks post-infection (Fig. 2). To determine if the multiply infection/cured mice additionally were able to clear infection throughout the body (not just at the site of initial infection), mice were immunosuppressed and then reassessed for parasites in the blood and tissues using microscopy (Fig. 2C, E, G). As expected both the continuously infected/challenged and the naïve/challenged mice had detectable parasitaemias following immunosuppression. However even mice submitted to three rounds of infection and cure failed to prevent the establishment of the infection following footpad challenge. The failure to prevent reinfection following one or two rounds of infection/cure is also evident from the fact that mice challenged with a low dose $\left(10^{3}\right)$ of $T$. cruzi i.p. show very low levels of CD127 expression on T. cruzi-specific T cells, indicative of a persistent antigen presence (Fig. 3A). 

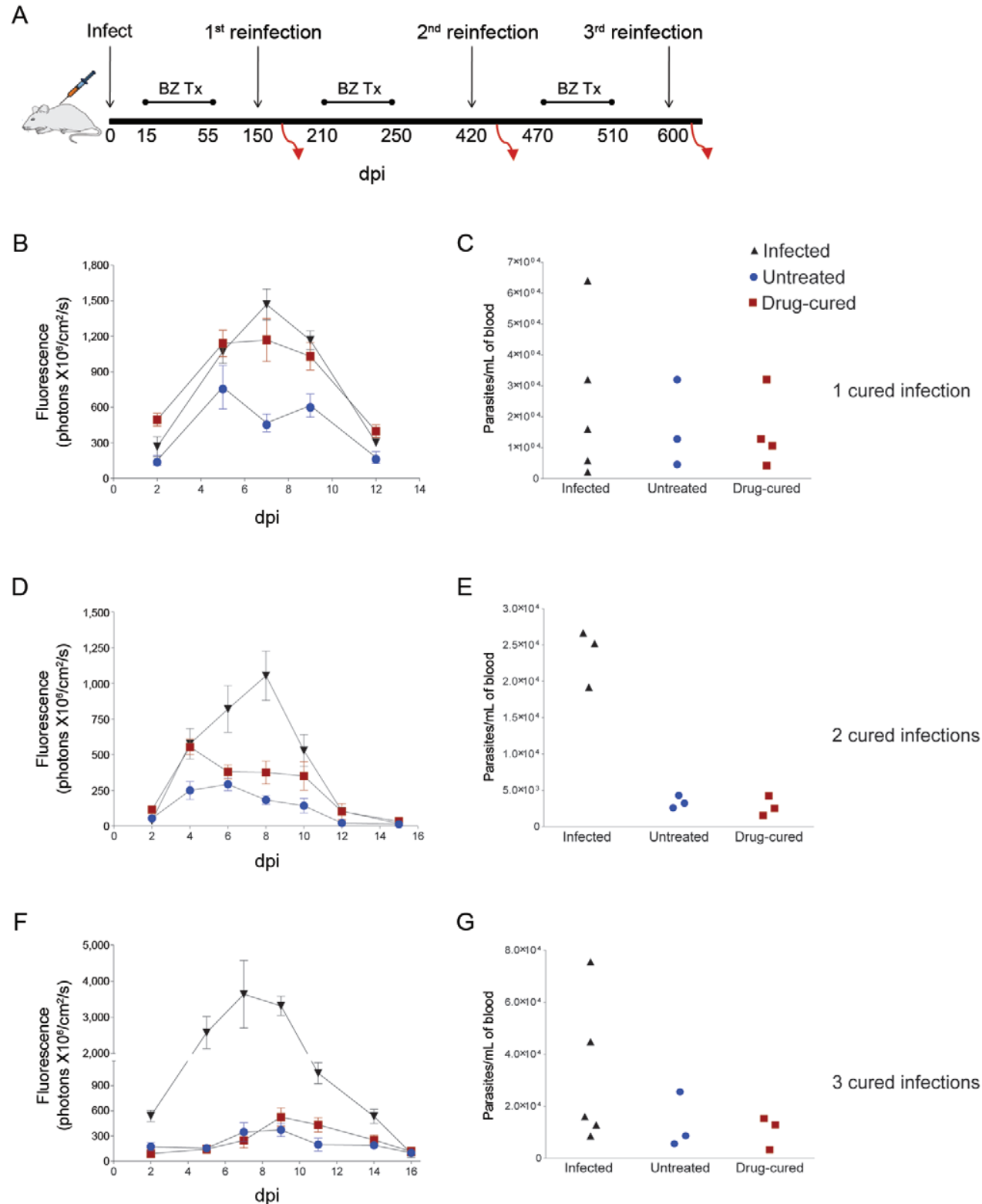

Fig. 2: enhanced infection control acquired after three cured infections. A: schematic representation of infection, treatment and reinfection. Red arrows indicate points at which a subset of mice was immunosuppressed to assess infection status (B, D, F). Parasite load at the site of the infection assessed by quantification of the fluorescent signal from mice primarily infected with $1 \times 10^{3} \mathrm{CL}$ wild-type parasites that underwent one, two or three cured infections and their untreated counterparts submitted to challenged in the hind foot pads with $2.5 \times 10^{5}$ Trypanosoma cruzi tdTomato trypomastigotes (C, E, G). Parasitaemias in mice described in B, D and F at $\sim 15$ days after administration of the immunosuppressant cyclophosphamide; BZ: benznidazole; dpi: days post-infection.

Although immunity induced by cure of an initial infection is generally the standard against which vaccines are measured, there are multiple reasons why the infection/cure protocol used herein failed to provide sterilising immunity. To exclude the possibility that immune effectors might not be generated, or are lost after BZ-induced cure, we monitored effector functions in $T$. cruzi-specific $\mathrm{T}$ cells during the infection cure cycle. The boost in the frequency of T. cruzi-specific T cells in cured mice following each round of reinfection is indicative of sustained and competent $\mathrm{T}$ cell memory following infection cure (Fig. 3B). Although the overall frequency of T. cru- $z i$-specific $\mathrm{CD} 8^{+} \mathrm{T}$ cells decreases, as expected following infection cure (Bustamante et al. 2008) (Figs 3B, 4A), the quality of these memory $\mathrm{T}$ cells is evidenced by their in vivo cytolytic activity (Fig. 4B) and ex vivo cytokine production in response to T. cruzi epitopes (Fig. 4C). Thus the failure of the infection cure protocol to provide protection is not due to the absence of an inducible effector $\mathrm{T}$ cell population that could be recalled upon challenge.

The argument could be made that the immunity induced by the infection/cure protocol used here induces an insufficient and/or poorly targeted immune response. The enhanced protection conveyed by active infection 
A

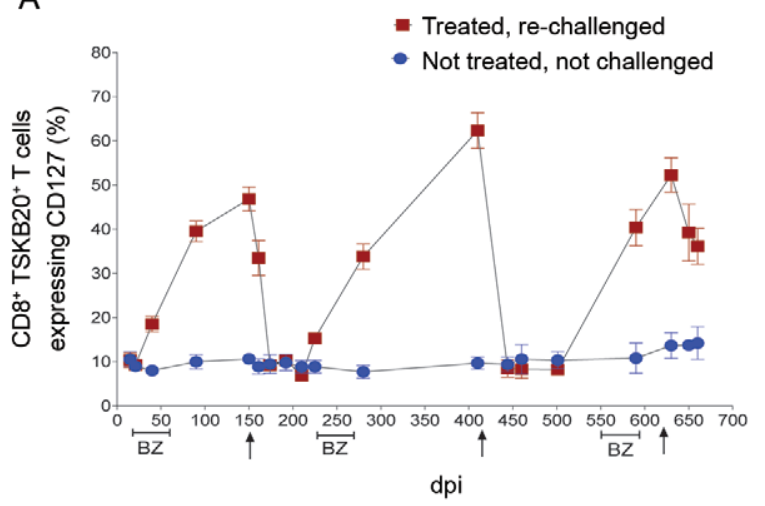

B

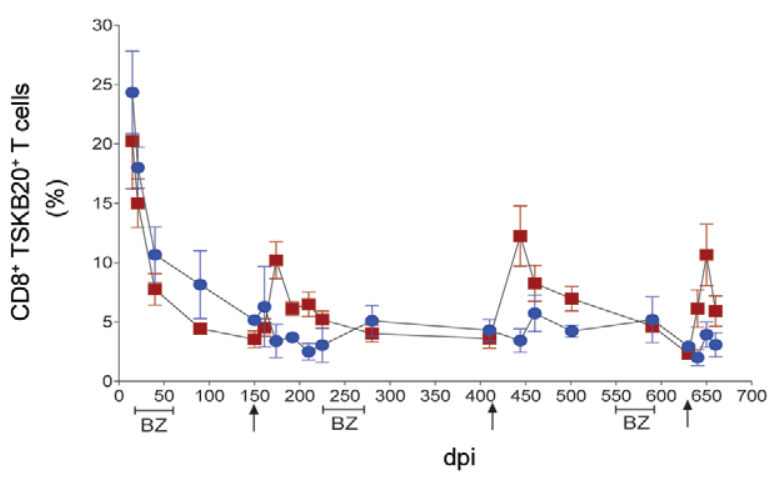

Fig. 3: parasite-specific memory $\mathrm{T}$ cell responses in mice undergoing multiple rounds of infection and cure. A: expression of the memory maintenance marker CD127 in blood on CD8 ${ }^{+}$TSKB20-tetramer $^{+} \mathrm{T}$ cells from infected mice that underwent three cured infections and their untreated counterparts; B: the MHC-peptide tetramer of the immunodominant TSKB20/Kb epitope was used to detect Trypanosoma cruzi-specific $\mathrm{CD}^{+} \mathrm{T}$ cells in the blood of mice described above; BZ: benznidazole; dpi: days post-infection.

relative to cured infection evident in the footpad challenge model might suggest that higher number of parasite-specific $\mathrm{T}$ cells, effector $\mathrm{T}$ cells, rather than memory $\mathrm{T}$ cells or effector mechanisms not measured in these assays, may be required for optimal infection control. However it is apparent in other studies (Bustamante et al. 2002, 2007) that superinfection (a "new" infection in an already infected host) is not uncommon in the case of $T$. cruzi, indicating that simply having more effector $\mathrm{T}$ cells (as is the case during an active infection) rather than memory $T$ cells (in cured infection) is not sufficient to prevent establishment of "new" infections. Thus, immunity induced by prior infection, whether cured or not, is insufficient to prevent reinfection.

We believe that the results of several recent studies shed some light on the failure of active infection and the infection/cure protocol used here, as well as other vaccine protocols in $T$. cruzi and may be informative with respect to defining the overall likelihood of developing competent prophylactic vaccines for $T$. cruzi infection. For the immune system to prevent the establishment of $T$. cruzi infection, the potentially protective response must either absolutely prevent parasites from infecting host cells, thus halting the replication of $T$. cruzi before it can begin or must rapidly identify and kill infected cells before the initial round of parasite replication can be completed (and the resulting expanded population of parasites spreads throughout the body).

With respect to the preventing host cell entry, the passive transfer of high-titre antibodies can reduce parasite loads (Franchin et al. 1997), presumably through the ability to target extracellular trypomastigotes for destruction by complement or phagocytic cells, or to block the ability of these parasites to invade host cells. However this approach has not been demonstrated to actually prevent infection and the antibodies produced during the course of acute and chronic infections, although contributing to immune control of the infection (Kumar \& Tarleton 1998) clearly lack the ability to prevent the constant reinfection of host cells that maintains the infection. So the ability to generate a targeted antibody response sufficient to block $100 \%$ of invading parasites seems questionable - certainly no previously tested method has.

Investigation of the early events in the generation of parasite-specific T cell responses in T. cruzi have provided some insights into how the naïve as well as the primed immune system deals with $T$. cruzi. Despite the strength and the focus of the $\mathrm{CD}^{+}$cells generated in $T$. cruzi infection, this response is nevertheless extremely slow in developing (Padilla et al. 2009). This delay is evident in the initiation of proliferation and development of effector function in parasite-specific $\mathrm{T}$ cells, as well as in the recruitment of leukocytes into the draining lymph nodes. These observations suggest initially $T$. cruzi is infection occurs "under the radar", failing to trigger significant recognition by pathogen sensors on with innate and adaptive immune responses depend. We have attributed this clandestine infection process by $T$. cruzi to two factors: (i) the mechanism of cell invasion by T. cruzi that occurs without significant host cell damage and thus with little release of damage associated molecular patterns (DAMPs) and (ii) the absence of significant pathogenassociated molecular patterns (PAMPs) in the invading $T$. cruzi trypomastigotes. These conclusions are supported by the observation of the delayed generation of responses, the timing of which follows closely the destruction of host cells (with the consequent release of DAMPs) during the exit of parasites at four-five dpi. Additionally, initiation of anti-T. cruzi $\mathrm{T}$ cell responses can be accelerated and enhanced by supplying bona fide PAMPs with the infection, either by administration of ligands for TLR2 and TLR9 coincident with infection (Padilla et al. 2009) or by endogenous expression of classical bacterial PAMPs by T. cruzi (Kurup \& Tarleton 2013).

The insufficiency of T. cruzi in triggering of innate immune sensors not only delays the initiation of responses in newly infected hosts, but also has a long-lasting impact on the ultimate course of T. cruzi infection. Infections with $T$. cruzi lines expressing Salmonella flagellin, a well-studied bacterial PAMP, control the infection better, exhibit much-reduced pathogen load and evidence of cure in some cases (Kurup \& Tarleton 2013). The implications of these results for the potential of vaccines to prevent $T$. cruzi infection are substantial. First, if T. cruzi can avoid eliciting an inflammatory response - at least 
A

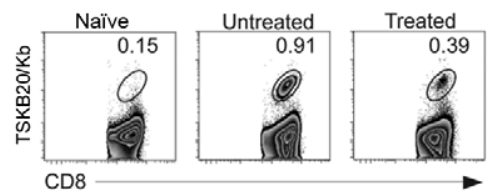

B

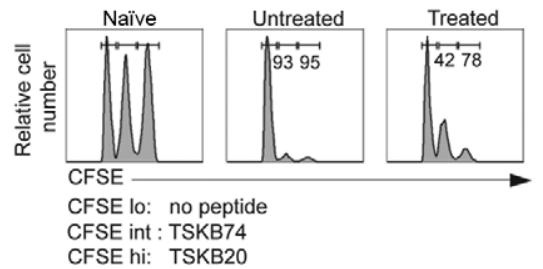

C

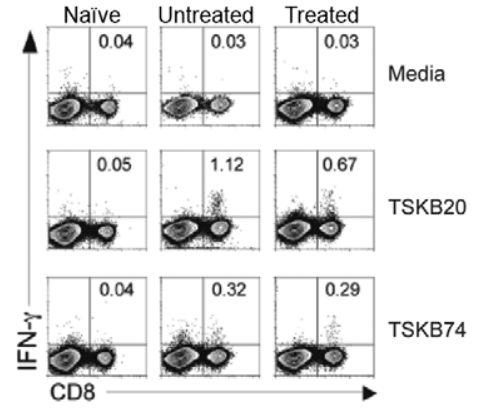

Fig. 4: functional effector T cell responses in Trypanosoma cruzi-infected and cured mice in the late stages of the infection. A: frequency of TSKB20/ $\mathrm{Kb}^{+} \mathrm{T}$ cells in the spleens of untreated or treated mice 615 days post-infection (dpi) (560 days post-treatment); B: in vivo cytotoxic T lymphocyte activity on TSKB20 and TSKB74-pulsed targets $16 \mathrm{~h}$ after transfer to naïve, untreated or treated $T$. cruzi-infected mice (615 dpi). Data are from representative individual mice. Numbers above the peaks are percentage of specific lysis calculated as described in the Material and Methods; C: intracellular cytokine staining for interferon (IFN)- $\gamma$ production by $\mathrm{CD}^{+} \mathrm{T}$ cells with or without stimulation with TSKB20 peptide in representative mice at $400 \mathrm{dpi}$. Numbers represent the percentage of $\mathrm{CD} 8^{+} \mathrm{T}$ cells producing IFN- $\gamma$.

until after completion of the first round of replication in and release from host cells - then no level of pre-existing $\mathrm{T}$ cell immunity (i.e., vaccine-induced immunity) will be able to prevent establishment of the infection in a newly infected host or quickly extinguish that infection once it is established. Second, each time T. cruzi invades cells in a "new" site in an infected host (e.g. a site lacking primed effector cells) parasite proliferation would also be expected to proceed there unfettered until the inflammatory signals generated by the destruction of the host cell recruit these effector cells beginning four-five days later (upon completion of the replication cycle). Indeed this trend is apparent in the footpad infection assays shown in Fig. 2; for the one and two-time infected/cured mice, as well as early infection in the continuously infected mice, there is essentially no control of parasite load detected in the infection site until after day 4 of infection.
A second potential complicating factor in immunity to $T$. cruzi and vaccine development is the targets of these immune responses. The major focus of both cellular and humoral immune responses in T. cruzi infection are proteins encoded by large gene families. With respect to $\mathrm{CD} 8^{+} \mathrm{T}$ cell responses, epitopes encoded by the ts family of genes are strongly immunodominant (Martin et al. 2006). The high variability (the ts family is composed of $>3,000$ individual genes/gene fragments in the T. cruzi CL Brener genome) and the strain-specific variation and recombinational potential of these genes (P Weatherly et al., unpublished observations) makes the ts proteins moving targets for immune responses and thus a relatively poor choice as vaccine targets. Furthermore, we have recently reported that ts epitopes are not effectively presented by infected host cells until 24-48 h after host cell infection. In contrast, other nonvariant but sub-dominant immune targets from flagellar proteins are detected by $\mathrm{T}$ cells within $6 \mathrm{~h}$ after host cell invasion (Kurup \& Tarleton 2014). Thus the normal T cell response to $T$. cruzi is fixated on a set of molecules that are highly variable and that are not expressed until late in the cycle of host cell infection - a poor "choice" for the effective elimination of infected host cells.

Collectively, the data presented in these studies and previously reported suggest the following scenario of events in T. cruzi infection. Infection of naïve, previously infected or persistently infected hosts, or in new sites within an infected host, occurs with minimal triggering of innate immune sensors, thus allowing the establishment of T. cruzi in these hosts/sites. T cell responses are generated and local inflammation is induced only after completion of a round of parasite replication and host cell destruction, which releases DAMPs and possibly PAMPs. The anti-T. cruzi $\mathrm{T}$ cells that are normally induced in the infection target primarily highly variant proteins that are not effectively presented on host cells until late in the host cell infection process. Thus $\mathrm{T}$ cells that are induced by infection or vaccination are both not initially recruited to sites of infection and when they ultimately get to these sites, they do not have the specificities that allow them to rapidly identify infected host cells early in the infection process. Vaccination could be used to redirect $\mathrm{T}$ cell responses to nonvariant antigens, such as those encoded in flagellar proteins - this process has been shown to have some merit (Kurup \& Tarleton 2014). However it is difficult to see how a vaccination can overcome the issue of the relatively silent invasion process used by $T$. cruzi. The best one might be able to hope for in an anti-T. cruzi vaccine is to enhance the rate of parasite clearance and reduce the time required to and frequency of cure of the infection - but not prevent the infection. While this would be an improvement, whether that is sufficient to justify a vaccine development program is uncertain.

The studies discussed here present the possibility that development of an effective prophylactic vaccine for $T$. cruzi may not be feasible. The incredible impact of vaccines in curbing deaths due to infectious agents notwithstanding, it may just be that there are infections that will allude protection by prophylactic vaccination and Cha- 
gas disease may be among these. A prophylactic vaccine is only one a number of vaccination modalities. Others have promoted the idea of therapeutic vaccines (Dumonteil et al. 2012). However these would likely suffer from the same problems as described here for prophylactic vaccines, as well as the concern of their utility given the availability of therapeutic drugs that can be highly effective, despite their variability and toxicities. Because T. cruzi infection is zoonotic and the fact that companion animals contribute significantly to its transmission to humans, transmission blocking vaccines that reduce pathogen load (without having to necessary eliminate the infection) have some promise. Lastly, related to the effectiveness of drugs to cure T. cruzi infection - as done in this study - it is important to note that hosts cured of T. cruzi infection are susceptible to reinfection. So the use and development of better methods of transmission control will continue to be a necessity if the impact of $T$. cruzi infection in humans is to be reduced.

\section{ACKNOWLEDGEMENTS}

To the present and former member of the Tarleton Research Group, for contributions, data discussed and the ideas expressed.

\section{REFERENCES}

Bertocchi GL, Vigliano CA, Lococo BG, Petti MA, Viotti RJ 2013. Clinical characteristics and outcome of 107 adult patients with chronic Chagas disease and parasitological cure criteria. Trans $R$ Soc Trop Med Hyg 107: 372-376.

Bustamante JM, Bixby LM, Tarleton RL 2008. Drug-induced cure drives conversion to a stable and protective $\mathrm{CD} 8^{+} \mathrm{T}$ central memory response in chronic Chagas disease. Nat Med 14: 542-550.

Bustamante JM, Craft JM, Crowe BD, Ketchie SA, Tarleton RL 2014. New, combined and reduced dosing treatment protocols cure Trypanosoma cruzi infection in mice. J Infect Dis 209: 150-162.

Bustamante JM, Novarese M, Rivarola HW, Lo Presti MS, Fernandez AR, Enders JE, Fretes R, Paglini-Oliva PA 2007. Reinfections and Trypanosoma cruzi strains can determine the prognosis of the chronic chagasic cardiopathy in mice. Parasitol Res 100: 1407-1410.

Bustamante JM, Rivarola HW, Fernandez AR, Enders JE, Fretes R, Palma JA, Paglini-Oliva PA 2002. Trypanosoma cruzi reinfections in mice determine the severity of cardiac damage. Int $J$ Parasitol 32: 889-896.

Canavaci AM, Bustamante JM, Padilla AM, Perez Brandan CM, Simpson LJ, Xu D, Boehlke CL, Tarleton RL 2010. In vitro and in vivo high-throughput assays for the testing of anti-Trypanosoma cruzi compounds. PLoS Negl Trop Dis 4: e740.

Collins MH, Craft JM, Bustamante JM, Tarleton RL 2011. Oral exposure to Trypanosoma cruzi elicits a systemic CD8 T cell response and protection against heterotopic challenge. Infect Immun 79: 3397-3406.
Cummings KL, Tarleton RL 2003. Rapid quantitation of Trypanosoma cruzi in host tissue by real-time PCR. Mol Biochem Parasitol 129: 53-59.

Dias JC, Dias E, Martins-Filho OA, Vitelli-Avelar D, Correia D, Lages E, Prata A 2008. Further evidence of spontaneous cure in human Chagas disease. Rev Soc Bras Med Trop 41: 505-506.

Dumonteil E, Bottazzi ME, Zhan B, Heffernan MJ, Jones K, Valenzuela JG, Kamhawi S, Ortega J, Rosales SP, Lee BY, Bacon KM, Fleischer B, Slingsby BT, Cravioto MB, Tapia-Conyer R, Hotez PJ 2012. Accelerating the development of a therapeutic vaccine for human Chagas disease: rationale and prospects. Expert Rev Vaccines 11: 1043-1055.

Franchin G, Pereira-Chioccola VL, Schenkman S, Rodrigues MM 1997. Passive transfer of a monoclonal antibody specific for a sialic aciddependent epitope on the surface of Trypanosoma cruzi trypomastigotes reduces infection in mice. Infect Immun 65: 2548-2554.

Francolino SS, Antunes AF, Talice R, Rosa R, Selanikio J, de Rezende JM, Romanha AJ, Dias JC 2003. New evidence of spontaneous cure in human Chagas disease. Rev Soc Bras Med Trop 36: 103-107.

Kumar S, Tarleton RL 1998. The relative contribution of antibody production and $\mathrm{CD} 8^{+} \mathrm{T}$ cell function to immune control of Trypanosoma cruzi. Parasite Immunol 20: 207-216.

Kurup SP, Tarleton RL 2013. Perpetual expression of PAMPs necessary for optimal immune control and clearance of a persistent pathogen. Nat Commun 4: 2616.

Kurup SP, Tarleton RL 2014. The Trypanosoma cruzi flagellum is discarded via asymmetric cell division following invasion and provides early targets for protective $\mathrm{CD} 8\left(^{+}\right) \mathrm{T}$ cells. Cell Host Microbe 16: 439-449.

Martin DL, Weatherly DB, Laucella SA, Cabinian MA, Crim MT, Sullivan S, Heiges M, Craven SH, Rosenberg CS, Collins MH, Sette A, Postan M, Tarleton RL 2006. CD8 ${ }^{+}$T-cell responses to Trypanosoma cruzi are highly focused on strain-variant transsialidase epitopes. PLoS Pathog 2: e77.

Padilla AM, Simpson LJ, Tarleton RL 2009. Insufficient TLR activation contributes to the slow development of $\mathrm{CD} 8^{+} \mathrm{T}$ cell responses in Trypanosoma cruzi infection. J Immunol 183: 1245-1252.

Quijano-Hernandez I, Dumonteil E 2011. Advances and challenges towards a vaccine against Chagas disease. Hum Vaccin 7: 1184-1191.

Rappuoli R, Pizza M, Del Giudice G, de Gregorio E 2014. Vaccines, new opportunities for a new society. Proc Natl Acad Sci USA 111: 12288-12293.

Tarleton RL 2007. Immune system recognition of Trypanosoma cruzi. Curr Opin Immunol 19: 430-434.

Tarleton RL 2013. The role of immunology in combating Trypanosoma cruzi infection and Chagas disease. Rev Esp Salud Publica 86: 33-39.

Vazquez-Chagoyan JC, Gupta S, Garg NJ 2011. Vaccine development against Trypanosoma cruzi and Chagas disease. Adv Parasitol 75: 121-146. 